\title{
Clinical profile and outcome of COVID-19 in haematological malignancies: experience from tertiary care centre in India
}

\author{
Anshul Gupta $^{1} \cdot$ Nihar Desai $^{1} \cdot$ Sanjeev $^{1} \cdot$ Priyanka Chauhan $^{1} \cdot$ Soniya Nityanand ${ }^{1} \cdot$ Zia Hashim $^{2} \cdot$ Mansi Gupta $^{2}$
}

Received: 22 July 2021 / Accepted: 16 August 2021 / Published online: 24 September 2021

(c) The Author(s), under exclusive licence to Springer-Verlag GmbH Germany, part of Springer Nature 2021

\begin{abstract}
There is limited evidence on various clinical aspects of SARS-CoV-2 infection in patients with haematological cancers. The risk factors, prognosis, and outcome of patients with haematological cancers with coexistent COVID-19 need to be explored in different subsets of population. A single-institutional prospective observational study was conducted at a tertiary level medical institute in North India. The clinical details of the recruited patients having haematological malignancies and diagnosed with COVID-19 between 15 March 2020 and 31 May 2021 were prospectively collected through the electronic patient database system. The outcomes with respect to 28-day and 56-day mortality and the associated risk factors for prognostication were analysed. Of the 5750 hospital admissions (inpatient and day-care) during the study period, two hundred and forty-two patients (4.2\%) were diagnosed with COVID-19. Acute leukaemia was the most common haematological malignancy, seen in 117 (48.3\%) patients. Eighty-nine (36.8\%) patients had moderate-to-severe COVID-19 while 153 (63.2\%) patients presented with mild infection. The 28-day and 56-day mortality rates in our cohort were $13.3 \%$ and $19.8 \%$ respectively. Amongst the risk factors associated with poor outcome, the severity of COVID-19 (HR =1.8, 95\% CI 1.16-10.35; $p=0.04)$, presence of secondary infection $(\mathrm{HR}=2.1,95 \% \mathrm{CI} 2.45-21.3 ; p=0.023)$, and need for invasive mechanical ventilation $(\mathrm{HR}=2.3,95 \%$ CI 1.8-18.43; $p=0.01$ ) were prognostically significant on multivariate log rank analysis. The risk of SARS-CoV-2 infection does not increase with haematological malignancies; however, the outcome remains poor in patients with severe COVID-19, requirement of invasive mechanical ventilation, and pre-existing bacterial/fungal infection at presentation.
\end{abstract}

Keywords COVID-19 $\cdot$ Haematological malignancies $\cdot$ Risk factors $\cdot$ Mortality

Mansi Gupta

mansi1712@gmail.com

Anshul Gupta

anshulhaemat@gmail.com

Nihar Desai

nihar89@gmail.com

Sanjeev

drsanjeev_ranchi@gmail.com

Priyanka Chauhan

priyanka.chauhan1785@gmail.com

Soniya Nityanand

soniy_nityanand@yahoo.com

Zia Hashim

ziasgpgi@gmail.com

1 Department of Hematology, Sanjay Gandhi Post Graduate Institute of Medical Sciences, Raebareli Road, Lucknow, U.P. 226014, India

2 Department of Pulmonary Medicine, Sanjay Gandhi Post Graduate Institute of Medical Sciences, Raebareli Road, Lucknow, U.P. 226014, India

\section{Introduction}

The coronavirus disease 2019 (COVID-19) emerged as one of the most devastating public health emergencies of international concern in recent times. It is known to be caused by the novel severe acute respiratory syndrome coronavirus-2 (SARS-CoV-2) virus. As of 20 July 2021, a total of 190,671,330 confirmed cases of COVID-19 along with $40,98,758$ fatalities have been officially recorded throughout the world [1]. COVID-19 manifests as a wide spectrum of clinical scenarios including asymptomatic cases, mildly symptomatic febrile viral illness, pneumonia, acute respiratory distress syndrome (ARDS), cytokine release syndrome (CRS), multi-organ dysfunction syndrome (MODS), etc. $[2,3]$. The mortality rates due to COVID-19 vary greatly among different countries due to heterogeneity in the susceptible population, infection rates, and the available healthcare infrastructure and support. However, the pooled data from several nations have shown that certain high-risk 
factors like hypertension, diabetes, cardiac diseases, cancers, age $>60$ years, ethnicity, etc., are uniformly associated with poorer outcomes $[4,5]$.

One of the most vulnerable sub-groups for COVID-19 could undoubtedly be the patients with various haematological malignancies undergoing treatment with chemotherapy. These patients are immunosuppressed due to not only their underlying disease but also the ongoing treatment. This generates a significant concern about the heightened risk of COVID-19-related morbidity and mortality in such patients [6]. To add to the complexity, all haematological malignancies do not pose the same risk for severe COVID-19. As per the available evidence, acute leukaemia and bone marrow failure syndrome appear to be at the highest risk of developing severe manifestations of COVID-19 [6]. A recently published meta-analysis by Abi et al., including 3377 patients of haematologic malignancies with COVID-19, showed that age $>60$ years, non-white population, and need for ICU admission carry a high risk of mortality. However, they could not demonstrate any association of recent systemic chemotherapy with increased risk of mortality. It was therefore concluded that most patients with haematologic malignancies survive, despite acquiring SARS-CoV-2 infection [7]. Some of the recent registry-based studies which include the COVID-19 and Cancer Consortium (CCC19), the UK Coronavirus Cancer Monitoring Project (UKCCMP), and the American Society of Haematology (ASH) Research Collaborative group have also emerged to answer many unanswered queries related to SARS-CoV-2 infection in patients with haematological malignancies.

Having already faced two devastating COVID-19 waves during the ongoing pandemic, with 31 million cases and 0.4 million fatalities as of 20 July 2021, India is currently the second most affected country in the world [1]. Due to the wide heterogeneity in population demographics, SARS-CoV-2 infection-related medical care, and haematooncology treatment practices offered at different hospitals across the Indian sub-continent, it becomes vital to analyse COVID-19-related outcomes in this subgroup of patients. Hence, this prospective study was planned with an objective to determine the outcomes related to SARS-CoV-2 infection in patients of haematological malignancies with respect to mortality as well as the risk factors associated with prognosis of the disease.

\section{Material and methods}

A single-institution prospective observational study was conducted at a tertiary-level medical institute in North India. The study cohort included patients with haematological malignancies who were either newly diagnosed or undergoing treatment/follow-up with the Department of
Haematology at our institute and had been diagnosed with SARS-CoV-2 infection between 15 March 2020 and 31 May 2021. The study was performed after obtaining approval from the institutional ethics committee (IEC no. 2020-5EMP-118), and a written informed consent from all the recruited patients. Patients meeting the inclusion criteria were recruited irrespective of the severity of COVID-19, setting of treatment (home isolation, outpatient treatment, or hospitalisation), and the remission status of the underlying haematological cancer. As per the institutional protocol, patients who were undergoing chemotherapy were screened for SARS-CoV-2 infection irrespective of any disease symptoms prior to OPD consultation, day-care chemotherapy, and inpatient hospital admissions. The diagnosis of COVID-19 was made on positive qualitative real-time reverse transcriptase-polymerase chain reaction (qRT- PCR) done on nasal and/or oropharyngeal swab samples. The genomic surveillance of SARS-CoV-2 virus was not done in our study. This study prospectively included patients who got infected during the two pandemic waves of COVID-19 in India between 2020 and 2021.

The following patient details were recorded in the electronic patient database system: demographic profile, nature of comorbidities, severity of COVID-19, remission status, and baseline disease characteristics including details of ongoing therapy. The baseline laboratory-based evaluation in every COVID-19 patient included complete blood counts, coagulation profile, kidney function tests, liver function tests, quantitative d-dimer levels, C-reactive protein levels (CRP), serum ferritin levels, serum procalcitonin levels, serum lactate dehydrogenase (LDH) levels, and a digital chest X-ray. High-resolution CT (HRCT) scan of chest was only done for moderate-to-severe cases. Documentation of various prognostic factors like age, sex, comorbidities, type of hematologic cancer, inflammatory markers, COVID-19 severity, and the details of ongoing therapy for the malignancy, etc., was also done. Follow-up (in days) period was taken from the date of first positive qRT-PCR report for SARS-CoV-2 to the last follow-up date or the date of death. Patients had a planned follow-up period of 56 days (minimum follow-up period 28 days). The detailed flowchart for patient recruitment in the study has been shown in Fig. 1.

All patients diagnosed with COVID-19 were classified and managed with uniform therapy as per the standardised institutional protocol (based on the latest national guidelines for the treatment of COVID-19, Ministry of Health and Family Welfare, India) [8]. Patients with benign haematological conditions, lack of sufficient patient details or follow-up less than 28 days, and patients with radiological COVID-19 diagnosis along with a negative qRT-PCR status were excluded. We included only microbiologically confirmed cases of COVID-19 (qRT-PCR positive) in this study. Patients who were qRT-PCR negative but 
Total hospital admissions with hematological malignancies screened for COVID-19

infection during study period ( $1^{\text {st }}$ April 2020-31 ${ }^{\text {st }}$ May 2021)

Inpatient admissions-3420

Day Care admissions-2330

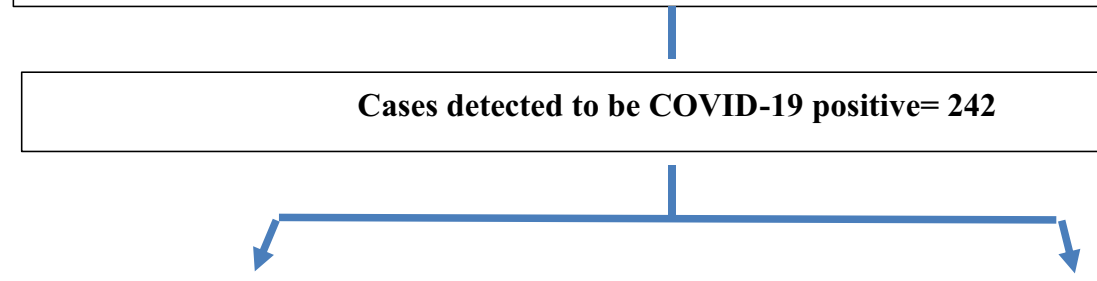

Mild infection $=153$

- Home isolation-53

- Isolation ward admission-100

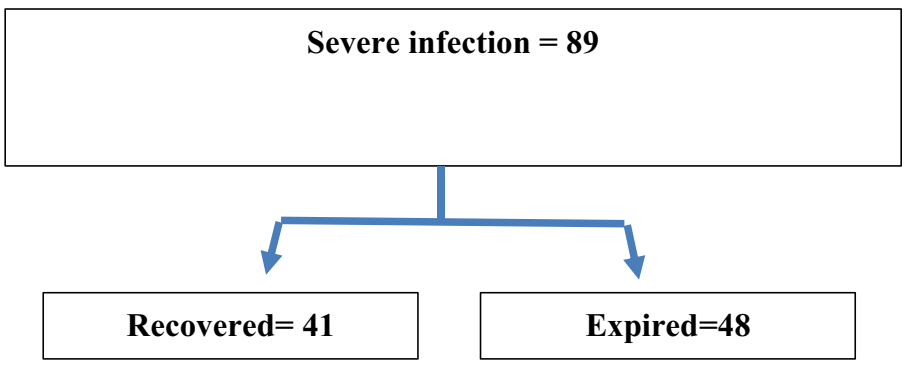

Fig. 1 Patient recruitment for the study

had an intermediate-high probability of COVID-19 diagnosis based on HRCT chest findings (CORADS 3-5) were excluded. All the recruited patients had a minimum follow-up of 28 days from the first qRT-PCR positive report for SARS-CoV-2; however, if any patient expired before this time point (day 28), it was included in the analysis and counted as an event.

\section{Statistical analysis}

Descriptive statistics were calculated using mean with standard deviation, or median with interquartile range. The categorical variables were represented using the frequencies with corresponding percentages. For categorical variables, Fisher's exact test or Chi-square tests were also used to analyse the differences in the distribution between individual parameters among various subsets of patients, while the Mann-Whitney $U$-test was used for the continuous variables. Outcomes were defined as death at day 28 and day 56 timepoints post qRT-PCR positivity, and cure was defined as two successive negative qRT-PCR reports more than $24 \mathrm{~h}$ apart. The univariate and multivariate analyses (Cox proportional hazard regression model) were also performed to consider each potential prognostic factor. SPSS statistical program (version 20) was used for all the statistical analysis and $p$-values less than 0.05 were considered statistically significant [9]. The cutoff date for data analysis was 31 May 2021.

\section{Results}

In the present study, 5750 hospital admissions (inpatient and day-care) of patients with haematological malignancies undergoing chemotherapy and/or admitted for supportive care were screened for SARS-CoV-2 infection during the study period. Two hundred forty-two patients $(4.2 \%)$ were found to be COVID positive by qRT-PCR test. The baseline demographic and clinical characteristics of our study cohort have been tabulated in Table 1. The median age of our cohort was 41 years (3-83 years) with a male to female ratio 1.92:1. Sixty-eight patients (28.1\%) in our cohort were $\geq 60$ years in age while 75 (31.0\%) patients belonged to the paediatric age group ( $<18$ years). Sixty-seven $(28.3 \%)$ patients had at least one co-morbidity at presentation, with hypertension being the most common co-morbidity. Acute leukaemia constituted the most common haematological malignancy comprising 117 (48.3\%) of the total COVID-19 patients followed by non-Hodgkin's lymphoma $(n=46 ; 19 \%)$ and multiple myeloma ( $n=36 ; 14.9 \%)$. Of the eight $(3.3 \%)$ patients who developed SARS-CoV-2 infection post-haematopoietic stem cell transplant, 06 patients developed severe disease. Almost $80 \%$ patients were receiving active treatment for their haematological cancers at the time of SARS-CoV-2 infection detection. The patients were further categorized into four subgroups based on the ongoing active therapies-chemotherapy alone in 124 (51.2\%) cases, chemo-immunotherapy in $39(16.1 \%)$ cases, oral targeted agents (imatinib, dasatinib, nilotinib, ibrutinib, lenalidomide) in 24 (9.9\%) patients, and 
Table 1 Baseline characteristics and demographic profile of haematological cases with COVID-19
Variable

All patients $(n=242)$

Age, years

Median (range)

41(3-83)

Age group, in years; $N(\%)$

$>80$
$60-80$
$40-60$
$18-39$
$<18$

$8(3.3)$

60(24.8)

$56(23.1)$

43(17.8)

$75(31)$

Sex; $N(\%)$

Female

83(34.3)

Male

159(65.7)

Coexisting co-morbidity (total); $N(\%)$

Cardiovascular disease

$68(28.1)$

Pulmonary disease

$38(15.7)$

Connective tissue

12(4.9)

Liver disease

Kidney disease

4(1.6)

32(13.2)

Diabetes

51(21.1)

Non- haematological cancers

04(1.6)

Type of haematological cancer; $N(\%)$

Acute myeloid leukaemia

32(13.2)

Acute lymphoblastic leukaemia

$85(35.2)$

Non-Hodgkin's lymphoma

46(19)

Hodgkin's lymphoma

$4(1.7)$

Multiple myeloma

Chronic myeloid leukaemia

36(14.9)

24(9.9)

CLL

$7(2.8)$

Post stem cell transplant

$8(3.3)$

Time from last chemotherapy for haematological malignancy; $N(\%)$

$$
<4 \text { weeks }
$$

$>4$ weeks

116(47.9)

126(52.1)

Status of haematological malignancy at COVID-19 diagnosis; $N(\%)$

Remission

94(38.8)

Active disease

$148(61.2)$

Blood group

A

$86(35.5)$

$84(34.7)$

AB

21(8.7)

$\mathrm{O}$

$51(21.1)$

COVID-19 symptoms at diagnosis; $N(\%)$

Asymptomatic

$08(3.3)$

Fever

209(86.4)

Myalgia

234(96.7)

Cough

159(65.7)

Dyspnea

87(35.9)

Diarrhoea

48(19.8)

Anti-cancer therapy; $N(\%)$

Treatment naïve

49(20.2)

Chemotherapy

124(51.2)

39(16.1)

24(9.9)

Oral targeted therapy

06(2.5)

Duration of COVID symptoms at diagnosis; $N(\%)$

$<7$ days

70 (28.9)

$\geq 7$ days 
post-allogenic stem-cell transplant immunosuppression in 06 $(2.5 \%)$ cases. The data was also analysed for the correlation between the ABO blood group and susceptibility to SARSCoV-2 infection/COVID-19. We found that the blood group A was the most common blood group in our COVID-19 positive cohort comprising 35.5\% cases followed by blood groups $\mathrm{B}, \mathrm{O}$, and $\mathrm{AB}$, constituting $34.7 \%, 21.1 \%$, and $8.7 \%$ of the SARS-CoV2 positive patients respectively (Table 1).

\section{SARS-CoV infection}

Of the total 242 SARS-CoV-2 positive patients in our cohort, $89(36.8 \%)$ patients had moderate-severe SARS-CoV-2 infection requiring ICU admission. Amongst the remaining $153(63.2 \%)$ patients with mild infection, $53(21.9 \%)$ were managed with symptomatic treatment in home isolation and recovered uneventfully. Myalgia was reported as the most common symptom at presentation, seen in 234 (96.7\%) cases followed by fever in 209 (86.4\%) SARS-CoV-2 positive cases. Eight (3.3\%) cases were asymptomatic at the time of SARS-CoV positivity and were detected incidentally during COVID-19 screening prior to chemotherapy (Table 1). Amongst the 08 haematopoietic stem-cell transplant (HSCT) recipients, 06 (75\%) patients had severe COVID-19 requiring ventilatory support, while two patients had a mild infection not requiring hospitalization. In our cohort, 158 (65.3\%) patients had COVID-19 symptom duration of $>7$ days. Eighty-four (34.7\%) of these patients had severe disease and required admission to the ICU. With respect to the disease status, $148(61.2 \%)$ patients had active haematological disease at the time of SARS-CoV-2 positivity and $49.3 \%$ of these patients presented with moderate-to-severe disease. Interestingly, only 16 (17\%) patients developed severe COVID-19 if their haematological cancer was under remission phase (Table 2). On analysis of the laboratory characteristics, the levels of d-dimer, procalcitonin, and C-reactive protein were significantly elevated in moderate-to-severe COVID-19 group as compared to patients with mild disease (Table 2). The median absolute lymphocyte count was found to be significantly low in the severe COVID-19 group $(p=0.04)$. All 89 patients $(36.8 \%)$ who were admitted to the ICU needed oxygen support (nasal prongs/facemask/nonrebreathing mask (NRBM)) throughout their stay. Of these ICU admissions, $51(57.3 \%)$ patients required ventilatory assistance by non-invasive ventilator and 38 (42.7\%) patients eventually required invasive mechanical ventilation. As part of the recommended institutional treatment plan for COVID19, patients with moderate-severe COVID-19 received remdesivir (anti-viral agent) protocol, while the mild cases $(n=153)$ received ivermectin and doxycycline. Also, all patients with moderate-severe COVID-19 received steroids, either dexamethasone $(0.05-0.2 \mathrm{mg} / \mathrm{kg} / \mathrm{day})$ or methylprednisolone $(0.5-1 \mathrm{mg} / \mathrm{kg} /$ day $)$ while $68(76.4 \%)$ of these cases received anticoagulation therapy with low molecular weight heparin/unfractionated heparin. The median time to qRT-PCR negativity was 21 days (14-56 days) in these 89 patients with ICU stay.

\section{Clinical characteristics of severe COVID infection cases}

Amongst the 89 cases requiring ICU admission, subgroup analysis was done for the survivors $(n=41)$ and the nonsurvivors $(n=48)$. We noticed that duration of COVID-19 symptoms $\geq 7$ days, quick Sequential Organ Failure Assessment (q-SOFA) score $\geq 2$, elevated inflammatory markers (CRP, serum ferritin, d-dimer, and procalcitonin), and preexisting bacterial/fungal infection at the time of COVID-19 diagnosis were associated with increased mortality as shown in Table 3.

\section{Survival analysis}

When the data was censored on 31 May 2021, 48 (19.8\%) patients had succumbed to their infection at a median followup period of 198 days (range: $14-402$ days). There were 53 events during the study period which included 48 severe SARS-CoV-2 infection-related deaths and 05 relapses of primary haematological malignancy ( 02 pre-B acute lymphoblastic leukaemia, 02 non-Hodgkin's lymphoma, 01 chronic lymphoblastic leukaemia). The 28-day and 56-day mortality rates of our cohort were $13.3 \%$ and $19.8 \%$ respectively.

\section{Univariate and multivariate analyses of the prognostic factors}

Univariate analysis of the potential prognostic factors predicting mortality in patients with SARS-CoV-2 infection showed that the presence of medical comorbidities (HR $=2.2,95 \%$ CI: $1.1-24.8 ; p=0.04)$, hospitalization after COVID-19 symptoms $>7$ days $(\mathrm{HR}=1.9,95 \% \mathrm{CI}$ $1.24-8.43 ; p=0.05)$, haematological disease status at the time of infection $(\mathrm{HR}=2.1,95 \% \mathrm{CI}(1.1-15.8) ; p=0.045)$, severity of SARS-CoV-2 infection $(\mathrm{HR}=2.8,95 \% \mathrm{CI}$ $3.5-12.6 ; p=0.03)$, presence of secondary infection at the time of presentation $(\mathrm{HR}=3.9,95 \%$ CI $2.78-16.8$; $p=0.01)$, and the requirement for invasive mechanical ventilation $(\mathrm{HR}=5.2,95 \%$ CI 3.45-26.76; $p=0.001)$ are associated with poor survival. Additionally, the patients who had absolute lymphocyte count (ALC) $\geq 1500$ cells/ $\mu 1$ had significantly better survival $(\mathrm{HR}=0.46,95 \% \mathrm{CI}$ $0.17-2.02 ; p=0.002)$. Of these variables, severity of COVID-19 (HR $=1.8,95 \%$ CI $1.16-10.35 ; p=0.04)$, presence of secondary infection at the time of presentation $(\mathrm{HR}=2.1,95 \%$ CI $2.45-21.3 ; p=0.023)$, and the 
Table 2 Clinical and lab characteristics of COVID-19 in haematological cases

\begin{tabular}{|c|c|c|c|c|}
\hline Variable & $\begin{array}{l}\text { All pts } \\
(n=242)\end{array}$ & Mild COVID $(n=153)$ & $\begin{array}{l}\text { Moderate/severe COVID } \\
(n=89)\end{array}$ & $p$ value \\
\hline $\begin{array}{l}\text { Age, years } \\
\text { Median (range) }\end{array}$ & $41(3-83)$ & $27.5(3-76)$ & $56(4-83)$ & 0.018 \\
\hline \multicolumn{5}{|l|}{ Type of haematological cancer; $N(\%)$} \\
\hline $\begin{array}{l}\text { Acute myeloid leukaemia } \\
\text { Acute lymphoblastic leukaemia } \\
\text { Non-Hodgkin's lymphoma } \\
\text { Hodgkin's lymphoma } \\
\text { Multiple myeloma } \\
\text { Chronic myeloid leukaemia } \\
\text { CLL } \\
\text { Post stem cell transplant }\end{array}$ & $\begin{array}{l}32(13.2) \\
83(35.2) \\
46(19) \\
4(1.7) \\
34(14.9) \\
24(9.9) \\
11(2.8) \\
8(3.3)\end{array}$ & $\begin{array}{l}24(75) \\
65(78.9) \\
16(33.3) \\
3(75) \\
22(66.6) \\
19(80) \\
2(28.5) \\
2(25)\end{array}$ & $\begin{array}{l}8(25) \\
18(21.1) \\
30(66.7) \\
1(25) \\
12(33.4) \\
5(20) \\
9(71.5) \\
6(75)\end{array}$ & $\begin{array}{l}0.12 \\
0.07 \\
0.23 \\
0.17 \\
0.09 \\
0.10 \\
0.11 \\
0.32\end{array}$ \\
\hline \multicolumn{5}{|l|}{ Duration of COVID symptoms; $N(\%)$} \\
\hline $\begin{array}{l}<7 \text { days } \\
\geq 7 \text { days }\end{array}$ & $\begin{array}{l}84(34.7) \\
158(65.3)\end{array}$ & $\begin{array}{l}60(85.7) \\
93(54)\end{array}$ & $\begin{array}{l}24(34.3) \\
65(37.8)\end{array}$ & 0.001 \\
\hline \multicolumn{5}{|c|}{ Time from last chemotherapy for haematological malignancy } \\
\hline $\begin{array}{l}<4 \text { weeks } \\
>4 \text { weeks }\end{array}$ & $\begin{array}{l}116(47.9) \\
126(52.1)\end{array}$ & $\begin{array}{l}73(62) \\
80(63.5)\end{array}$ & $\begin{array}{l}43(48.3) \\
46(51.7)\end{array}$ & 0.844 \\
\hline \multicolumn{5}{|c|}{ Status of haematological malignancy at COVID-19 diagnosis; $N(\%)$} \\
\hline $\begin{array}{l}\text { Remission } \\
\text { Active disease }\end{array}$ & $\begin{array}{l}94(38.8) \\
148(61.2)\end{array}$ & $\begin{array}{l}78(83.0) \\
75(50.7)\end{array}$ & $\begin{array}{l}16(17) \\
73(49.3)\end{array}$ & 0.009 \\
\hline \multicolumn{5}{|l|}{ Laboratory findings, median (IQR) } \\
\hline 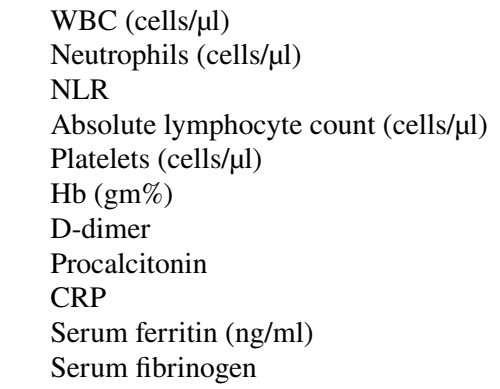 & $\begin{array}{l}5800(2925-10,625) \\
2150(1275-5550) \\
2.5(1.063-5.583) \\
1500(1200-4800) \\
116(42,000-1.64) \\
9.25(7.72-10.47) \\
368(221-887.5) \\
0.21(0.08-1.235) \\
17.5(10.25-41.75) \\
452(295-1446) \\
324(280-420)\end{array}$ & $\begin{array}{l}5350(2625--8975) \\
3150(1425-5800) \\
2.36(1.31-4.55) \\
2100(1600-4800) \\
135(48.5-1.80) \\
9.6(8.6-10.32) \\
287(190.75-666.75) \\
0.12(0.06-0.30) \\
12.5(9.5-24.25) \\
342(267-551.25) \\
300(268.7-380)\end{array}$ & $\begin{array}{l}8250(3000-33,825) \\
2100(1150-5450) \\
3.46(0.488-22.125) \\
1100(900-2100) \\
89(27-140) \\
8.25(6.87-10.55) \\
765(338.5-1900) \\
3.37(0.23-7.185) \\
46.5(22.75-130) \\
1700(577-3712) \\
393(321-472.5)\end{array}$ & $\begin{array}{l}0.163 \\
0.645 \\
0.591 \\
0.04 \\
0.093 \\
0.177 \\
0.001 \\
0.001 \\
0.005 \\
0.02 \\
0.09\end{array}$ \\
\hline \multicolumn{5}{|l|}{ Coexisting co-morbidity(total); $N(\%)$} \\
\hline $\begin{array}{l}\text { Cardiovascular disease } \\
\text { Pulmonary disease } \\
\text { Connective tissue } \\
\text { Liver disease } \\
\text { Kidney disease } \\
\text { Diabetes } \\
\text { Non-haematological cancers }\end{array}$ & $\begin{array}{l}68(28.1) \\
38(15.7) \\
12(4.9) \\
4(1.6) \\
32(13.2) \\
51(21.1) \\
04(1.6)\end{array}$ & $\begin{array}{l}28(41.2) \\
23(60.5) \\
10(83.3) \\
2(50) \\
16(50) \\
15(38.5) \\
01(25)\end{array}$ & $\begin{array}{l}40(58.8) \\
15(39.5) \\
2(16.7) \\
2(50) \\
16(50) \\
36(61.5) \\
03(75)\end{array}$ & $\begin{array}{l}0.07 \\
0.216 \\
0.985 \\
0.612 \\
0.475 \\
0.046 \\
0.443\end{array}$ \\
\hline \multicolumn{5}{|l|}{ Blood group } \\
\hline $\begin{array}{l}\mathrm{A} \\
\mathrm{B} \\
\mathrm{AB} \\
\mathrm{O}\end{array}$ & $\begin{array}{l}86(35.5) \\
84(34.7) \\
21(8.7) \\
51(21.1)\end{array}$ & $\begin{array}{l}55(64.0) \\
52(61.9) \\
13(61.9) \\
33(64.7)\end{array}$ & $\begin{array}{l}31(36.0) \\
32(38.1) \\
08(38.1) \\
18(35.3)\end{array}$ & $\begin{array}{l}0.09 \\
0.14 \\
0.12 \\
0.11\end{array}$ \\
\hline
\end{tabular}

requirement for invasive mechanical ventilation $(\mathrm{HR}=2.3$, 95\% CI 1.8-18.43; $p=0.01$ ) retained their prognostic significance with respect to overall survival (OS) on multivariate analysis as shown in Table 3 . We could not demonstrate any statistically significant correlation between the ABO blood group and the disease severity or the overall survival (OS) in the univariate analysis (Tables 2 and 4).

\section{Discussion}

We presented our experience of managing 242 patients of haematological malignancies infected with novel SARSCoV-2 virus, at a tertiary-level medical institute with a dedicated COVID-19 care hospital. All the recruited patients were uniformly treated based on a well-standardised COVID-19 treatment protocol. Being a novel virus, several 
Table 3 Clinical and lab characteristics of severe COVID-19 haematological cases

\begin{tabular}{|c|c|c|c|c|}
\hline Variable & $\begin{array}{l}\text { All pts } \\
(n=89)\end{array}$ & $\begin{array}{l}\text { Survivor } \\
(n=41)\end{array}$ & $\begin{array}{l}\text { Non-survivor } \\
(n=48)\end{array}$ & $p$ value \\
\hline \multicolumn{5}{|l|}{ Age, years } \\
\hline Median (range) & $56(4-83)$ & $58(4-80)$ & $52(15-83)$ & 0.96 \\
\hline \multicolumn{5}{|l|}{ Type of haematological cancer; $N(\%)$} \\
\hline $\begin{array}{l}\text { Acute myeloid leukaemia } \\
\text { Acute lymphoblastic leukaemia } \\
\text { Non-Hodgkin's lymphoma } \\
\text { Hodgkin's lymphoma } \\
\text { Multiple myeloma } \\
\text { Chronic myeloid leukaemia } \\
\text { CLL } \\
\text { Post stem cell transplant }\end{array}$ & $\begin{array}{l}8(25) \\
18(21.1) \\
30(66.7) \\
1(25) \\
12(33.4) \\
5(20) \\
9(71.5) \\
6(75)\end{array}$ & $\begin{array}{l}03(37.5) \\
07(38.9) \\
12(40) \\
01(100) \\
05(41.7) \\
04(80) \\
07(77.7) \\
02(33.3)\end{array}$ & $\begin{array}{l}05(62.5) \\
11(61.1) \\
18(60) \\
0 \\
07(58.3) \\
1(20) \\
02(22.2) \\
04(66.7)\end{array}$ & $\begin{array}{l}0.12 \\
0.29 \\
0.10 \\
0.21 \\
0.18 \\
0.11 \\
0.19 \\
0.17\end{array}$ \\
\hline \multicolumn{5}{|l|}{ Duration of COVID symptoms; $N(\%)$} \\
\hline $\begin{array}{l}<7 \text { days } \\
\geq 7 \text { days }\end{array}$ & $\begin{array}{l}24(27) \\
65(73)\end{array}$ & $\begin{array}{l}21(87.5) \\
20(30.8)\end{array}$ & $\begin{array}{l}03(21.5) \\
45(69.2)\end{array}$ & 0.04 \\
\hline \multicolumn{5}{|l|}{ ECOG performance status; $N(\%)$} \\
\hline $\begin{array}{l}<2 \\
\geq 2\end{array}$ & $\begin{array}{l}63(69.8) \\
26(29.2)\end{array}$ & $\begin{array}{l}31(49.2) \\
10(38.5)\end{array}$ & $\begin{array}{l}32(50.8) \\
16(61.5)\end{array}$ & 0.12 \\
\hline \multicolumn{5}{|l|}{ qSOFA score at ICU admission; $N(\%)$} \\
\hline $\begin{array}{l}<2 \\
\geq 2\end{array}$ & $\begin{array}{l}50(18.2) \\
39(81.8)\end{array}$ & $\begin{array}{l}33(66) \\
08(20.5)\end{array}$ & $\begin{array}{l}17(34) \\
31(79.5)\end{array}$ & 0.04 \\
\hline \multicolumn{5}{|l|}{ Time from last chemotherapy; $N(\%)$} \\
\hline $\begin{array}{l}<4 \text { weeks } \\
>4 \text { weeks }\end{array}$ & $\begin{array}{l}43(48.3) \\
46(51.7)\end{array}$ & $\begin{array}{l}23(53.5) \\
18(39.1)\end{array}$ & $\begin{array}{l}20(46.5) \\
28(60.9)\end{array}$ & 0.67 \\
\hline \multicolumn{5}{|c|}{ Status of haematological malignancy at COVID-19 diagnosis; $N(\%)$} \\
\hline $\begin{array}{l}\text { Remission } \\
\text { Active disease }\end{array}$ & $\begin{array}{l}16(18) \\
73(82)\end{array}$ & $\begin{array}{l}3(18.7) \\
38(52.1)\end{array}$ & $\begin{array}{l}13(81.3) \\
35(47.9)\end{array}$ & 0.07 \\
\hline \multicolumn{5}{|l|}{ Pre-existing co-infections; $N(\%)$} \\
\hline $\begin{array}{l}\text { Bacterial } \\
\text { Fungal }\end{array}$ & $\begin{array}{l}37(41.6) \\
16(18)\end{array}$ & $\begin{array}{l}06(16.2) \\
04(25)\end{array}$ & $\begin{array}{l}31(83.8) \\
12(75)\end{array}$ & \\
\hline $\begin{array}{l}\text { CT severity index at ICU admission; } N(\%) \\
\quad<10 \\
\quad 10-15 \\
16-20 \\
>20\end{array}$ & $\begin{array}{l}4(4.5) \\
53(59.1) \\
24(27.3) \\
8(9.0)\end{array}$ & $\begin{array}{l}4(100) \\
28(66) \\
8(41.7) \\
1(12.5)\end{array}$ & $\begin{array}{l}0 \\
25(34) \\
16(58.3) \\
7(87.5)\end{array}$ & - \\
\hline \multicolumn{5}{|l|}{ Laboratory findings, median (IQR) } \\
\hline $\begin{array}{l}\text { WBC } \\
\text { Neutrophils } \\
\text { NLR } \\
\text { Absolute lymphocyte count } \\
\text { Platelets } \\
\text { Hb } \\
\text { D-dimer } \\
\text { Procalcitonin } \\
\text { CRP } \\
\text { Serum ferritin } \\
\text { Serum fibrinogen }\end{array}$ & $\begin{array}{l}8250(3000-33,825) \\
2100(1150-5450) \\
3.46(0.488-22.125) \\
1100(900-2100) \\
89(27-140) \\
8.25(6.87-10.55) \\
765(338.5-1900) \\
3.37(0.23-7.185) \\
46.5(22.75-130) \\
1700(577-3712) \\
393(321-472.5)\end{array}$ & $\begin{array}{l}8400(3000-33,500) \\
2000(1600-3600) \\
0.5(0.25-17.0) \\
800(450-1500) \\
91(25-149) \\
9(7.7-10.7) \\
400(249-860) \\
0.25(0.12-4.24) \\
23(9-85) \\
970(507-1940) \\
393(301-467)\end{array}$ & $\begin{array}{l}8100(1000-34,800) \\
4100(200-5600) \\
8.5(3-27.5) \\
350(150-850) \\
85(48-137) \\
7.3(6.5-10.5) \\
1200(670-2200) \\
4.44(2.51-11.59) \\
128(96-186) \\
2492(725-6128) \\
392(355-490)\end{array}$ & $\begin{array}{l}0.87 \\
0.84 \\
0.05 \\
0.03 \\
0.92 \\
0.30 \\
0.02 \\
0.005 \\
0.001 \\
0.08 \\
0.81\end{array}$ \\
\hline \multicolumn{5}{|l|}{ Treatment; $N(\%)$} \\
\hline $\begin{array}{l}\text { Steroids } \\
\text { Remdesivir } \\
\text { Convalescent plasma } \\
\text { LMWH } \\
\text { Tociluzumab } \\
\text { Mechanical ventilation }\end{array}$ & $\begin{array}{l}89(100) \\
89(100) \\
40(45) \\
68(76.4) \\
8(9.0) \\
38(42.7)\end{array}$ & $\begin{array}{l}41(90.1) \\
41(100) \\
18(54.5) \\
38(72.7) \\
2(9.0) \\
02(18.2)\end{array}$ & $\begin{array}{l}48(100) \\
48(100) \\
22(36.3) \\
30(81.8) \\
6(9.0) \\
36(100)\end{array}$ & $\begin{array}{l}0.306 \\
0.84 \\
0.99 \\
0.611 \\
0.11 \\
0.001\end{array}$ \\
\hline
\end{tabular}


Table 4 Univariate and multivariate analyses of prognostic factors in COVID-19 infection with haematological malignancies

\begin{tabular}{|c|c|c|c|c|c|}
\hline \multirow[t]{2}{*}{ S. no } & \multirow[t]{2}{*}{ Variable } & \multicolumn{2}{|c|}{ Univariate analysis for OS } & \multicolumn{2}{|c|}{ Multivariate analysis for OS } \\
\hline & & $\mathrm{HR}(95 \% \mathrm{CI})$ & $p$ value & $\mathrm{HR}(95 \% \mathrm{CI})$ & $p$ value \\
\hline 1 & $\begin{array}{l}\text { Age } \\
<60 \text { years vs }>60 \text { years }\end{array}$ & $1.8(0.42-6.66)$ & 0.07 & - & - \\
\hline 2 & $\begin{array}{l}\text { Presence of } \geq 2 \text { medical comorbidities } \\
\text { Present vs absent }\end{array}$ & $2.2(1.1-24.8)$ & 0.04 & $1.2(0.55-5.37)$ & 0.12 \\
\hline 3 & $\begin{array}{l}\text { Time to hospital admission after COVID-19 symptoms } \\
<7 \text { days vs }>7 \text { days }\end{array}$ & $1.9(1.24-8.43)$ & 0.05 & $1.1(0.67-1.96)$ & 0.60 \\
\hline 4 & $\begin{array}{l}\text { Time from last chemotherapy } \\
<3 \text { weeks vs }>3 \text { weeks }\end{array}$ & $1.16(0.45-4.86)$ & 0.519 & - & - \\
\hline 5 & Absolute lymphocyte count at admission: $>1500$ vs $\leq 1500$ & $0.46(0.17-0.96)$ & 0.02 & $0.92(0.24-4.5)$ & 0.12 \\
\hline 6 & $\begin{array}{l}\text { Disease status at COVID-19 diagnosis } \\
\text { Remission vs non-remission }\end{array}$ & $2.1(1.1-5.8)$ & 0.045 & $1.15(0.23-5.68)$ & 0.09 \\
\hline 7 & $\begin{array}{l}\text { Type of chemotherapy for haematological malignancy } \\
\text { Highly myelosuppressive vs targeted therapy }\end{array}$ & $1.1(0.19-2.28)$ & 0.49 & - & - \\
\hline 8 & $\begin{array}{l}\text { Presence of secondary infection at the time of COVID - } 19 \text { diagnosis } \\
\text { Present vs absent }\end{array}$ & $3.9(2.78-16.8)$ & 0.01 & $2.1(2.45-21.3)$ & 0.023 \\
\hline 9 & $\begin{array}{l}\text { Severity of COVID-19 infection } \\
\text { Severe vs non-severe }\end{array}$ & $2.8(3.5-12.6)$ & 0.03 & $1.8(1.16-10.35)$ & 0.04 \\
\hline 10 & $\begin{array}{l}\text { Type of haematological malignancy } \\
\text { Aggressive vs indolent }\end{array}$ & $1.13(0.62-4.23)$ & 0.45 & - & - \\
\hline 11 & $\begin{array}{l}\text { Duration of ICU stay } \\
<14 \text { days vs }>14 \text { days }\end{array}$ & $1.23(0.23-3.46)$ & 0.41 & - & - \\
\hline 12 & $\begin{array}{l}\text { Mechanical ventilation } \\
\text { Yes vs No }\end{array}$ & $5.2(3.45-26.76)$ & 0.001 & $2.3(1.8-18.43)$ & 0.01 \\
\hline 13 & $\begin{array}{l}\text { ABO blood group } \\
\text { Blood group A vs non-A }\end{array}$ & $1.12(0.65-12.3)$ & 0.13 & - & - \\
\hline
\end{tabular}

issues like the disease course, optimal treatment strategies, and prognosis are largely unexplored in the subgroup of immunocompromised patients with haematological cancers. Due to severely supressed cellular and humoral immunity, these patients are theoretically at a greater risk of COVID19 [9-13]. In this study, we observed an overall prevalence rate of SARS-CoV-2 infection to be $4.2 \%$ in patients with haematological malignancies. Recently, He et al. in their series comprising 128 patients hospitalized for haematological cancers in Wuhan had reported case rates up to $10 \%$ (95\% CI: 6-17\%), which is higher than that reported in our series [14]. There is paucity of Indian data on COVID-19 prevalence rate in different haematological malignancies due to paucity of nationwide cancer registry-based surveys to address this issue [15]. Data from a few Italian surveys on haematological malignancies suggested low prevalence rates of COVID-19 in patients suffering from adult $\mathrm{Ph}+$ acute lymphoblastic leukaemia (0.4\%), chronic lymphocytic leukaemia $(0.5 \%)$, and chronic myeloid leukaemia $(0.17 \%)$ [16-18]. Similar to these results, our data also suggests that the presence of a haematological cancer does not increase the risk to acquire SARS-CoV-2 infection as such.

In our series, 153 (63.2\%) patients had mild disease at presentation requiring only symptomatic treatment with home isolation/short admission in isolation unit. Interestingly, none of these patients progressed to severe disease manifestations as well. In coherence with our results, the All-India Institutes of Medical Sciences (AIIMS) Haematology task force group also reported that $52.3 \%$ patients in their series had mild COVID-19 [15]. Additionally, in their series, $11.5 \%$ patients were completely asymptomatic at COVID-19 diagnosis, as compared to $3.3 \%$ patients reported in our series. However, contrary to the Indian data, the multicentre American Society of Haematology (ASH) collaborative research data hub reported mild disease in $31 \%$ patients while the rest had moderate-severe COVID-19 requiring ICU support [19]. Various factors like the ethnicity, social background, intensity of the immunosuppressive treatment, type of haematological cancer, and disease status at the time of COVID-19 diagnosis (remission vs. no remission) might have contributed to this difference in disease severity.

One of the key issues that needs to be answered is whether haematological malignancy predispose to COVID19. On one hand, Paul et al. recently reported that haematological cancers are not at increased risk of contracting COVID-19 and its prevalence is similar to that of general population, while on the other hand, He et al. from China showed that patients suffering from cancers are at twofold 
increased risk of getting infected with SARS-CoV-2 as compared to the native population [14]. Similarly, a United Kingdom (UK) population-based study reported that cancer patients had $60 \%$ increased risk of COVID-19 especially with age $>65$ years and male sex [20]. Investigators from China have shown that patients with cancer are prone to developing severe symptomatic disease with rapid progression as compared to general population (median 13 vs. 43 days; $p<0.001$ ) [21]. Similarly, Lattenist et al. had reported that patients with cancers were at higher risk of severe COVID-19, greater number of ICU admissions, invasive mechanical ventilation, and death, when compared with age- and sex-matched controls from population (39 vs. $8 \%$; $p=0.0003$ ) [22]. Thus, most of the studies are showing conflicting results and large country-wide cancer registry-based surveys are needed to answer this question.

Another important objective of our study was to determine the risk factors in Indian patients with haematological cancers that may have prognostic relevance in co-existent COVID-19 management. According to our results on multivariate analysis, the severity of SARS-CoV-2 infection at admission (severe vs. non severe), presence of secondary infection at the time of COVID-19 diagnosis, and the need for invasive mechanical ventilation were significantly associated with poor survival in our cohort. Some of the previous studies had reported that severe COVID-19 and the need for oxygen therapy were associated with increased mortality and poor outcome [23-25]. Interestingly, we noticed that patients who had pre-existing bacterial or fungal infection at presentation had extremely poor outcome. A total of $83.8 \%$ patients with documented bacterial sepsis (on cultures) expired following the superadded COVID-19 infection. Similarly, 12 out of 16 patients who had probable/ proven invasive pulmonary aspergillosis succumbed to their illness following COVID-19 infection. This highlights the fact that COVID-19 further worsens the clinical course due to hyperinflammatory host response and thereby increases the probability of mortality due to CRS and MODS. It has been documented earlier that the immune response of body to the damaged and dying alveolar cells is orchestrated by the release of signal molecules called danger-associated molecular patterns (DAMPs), which lead to an amplified inflammatory cascade and extensive local tissue injury [26]. In addition to these factors, presence of $\geq 2$ co-morbidities and time to hospitalization $\geq 7$ days were found to be relevant prognostic factors on univariate analysis; however, these could not reach the level of statistical significance on multivariate analysis probably due to small cohort size. Like our findings, a Spanish study group in their large populationbased study reported that the presence of $\geq 2$ co-morbidities is significantly associated with increased mortality risk [27]. However, contrary to our results, an Indian multicentre study did not demonstrate any such association of comorbidities to mortality risk [15]. We could not demonstrate any association of age $>60$ years with prognosis in our patients. This was possibly due to the wide range in age distribution, with almost two-thirds of patients presenting with a mild disease.

We have reported the day 28 and day 56 mortality rates to be $13.3 \%$ and $19.8 \%$ respectively in our study cohort. These are comparable to the day 28 mortality rates of $14.2 \%$ in the non-cancer COVID-19 patients $(n=5321)$ who were treated at our dedicated COVID-19 care hospital during the study period. However, since this comparison is not matched for age, sex, and comorbidity, it is difficult to draw meaningful conclusions. Yigenoglu and colleagues from Turkey had reported a case fatality rate of $13.8 \%$ in 740 cases of haematological malignancies with COVID-19 infection [28]. It is to be emphasized here that in cases with severe COVID19 , our mortality rate reached $53.9 \%$. However, Abi et al. reported the risk of death to be almost $35 \%$ in hospitalized patients of COVID-19 with haematological cancers (95\% CI, 28-39) [7]. In another Spanish study, Saurez et al. reported an all-cause mortality rate of $33 \%$ in their cohort comprising 697 haemato-oncology patients [27]. The high mortality rate in both these studies can be attributed to the higher number of cases with moderate-severe infection who were hospitalized for disease management similar to our findings.

The main strengths of our study are the prospective study design and uniform treatment of all COVID-19 patients with well-standardised institutional treatment protocol. However, being a single-institution study, the results of this study cannot be generalized to represent the outcome of COVID-19 in the subgroup of patients with haematological malignancies for the entire country. Prospective multi-institutional studies are the need of the hour to conclusively answer several questions which are still a dilemma to the treating physicians. There are many unresolved issues pertaining to optimisation of treatment strategies, prediction of treatment toxicities, and improvement in the cure rates in haemato-oncology patients with COVID-19 infection.

To conclude, our study has demonstrated that though the presence of any haematological cancer does not predispose an individual to acquire SARS-CoV-2 infection but once acquired, the outcome of SARS-CoV-2 infection is poor particularly in the subgroup with severe disease, late presentation ( $>7$ days of illness), and pre-existing bacterial/fungal infection at the time of COVID-19 diagnosis. An early diagnosis of COVID-19 through proper screening at each day care/OPD visit, and prompt hospitalization of moderate-severe disease in patients with haematological cancers, is the key to improve outcome.

Acknowledgements The study was carried out in Sanjay Gandhi post Graduate Institute of Medical Sciences, Lucknow, India. We acknowledge the contribution of clinical haematologist Dr Rajesh Kashyap for recruitment of patients. We also acknowledge the significant contribution of haematopathologists, Dr Ruchi Gupta, Dr. Dinesh Chandra, 
and Dr. Manish Kumar Singh. We thank all the resident doctors, and nursing staff working in the department, without whom this study was impossible.

Data availability statement The data that support the findings of this study are available on request from the corresponding author, Dr. Mansi Gupta.

\section{Declarations}

Conflict of interest The authors declare no competing interests.

Ethics approval The study has been approved by Institution Ethics Committee (IEC No. 2020-5-EMP-118).

Patient consent Written informed consent has been taken from all patients included in the study.

Permission to reproduce material from other sources This is the original research and all figures, diagrams, schemes, tables, and text in this manuscript are our own, original, unpublished work.

\section{References}

1. Coronavirus Update (Live): 9,63,04429 cases and 17,95178 deaths from COVID-19 virus pandemic - worldometer [Internet]. [last accessed on $20^{\text {th }}$ July 2021]. Available from: https://www.world ometers.info/coronavirus/\#countries

2. Siordia JA Jr (2020) Epidemiology and clinical features of COVID-19: a review of current literature. J Clin Virol 127:104357. https://doi.org/10.1016/j.jcv.2020.104357

3. Sahu KK, Kumar R (2020) Current perspective on pandemic of COVID-19 in the United States. J Fam Med Prim Care 9:17841791. https://doi.org/10.4103/jfmpc.jfmpc_424_20

4. Jordan RE, Adab P, Cheng KK (2020) Covid-19: risk factors for severe disease and death. BMJ 368:m1198. https://doi.org/10. 1136/bmj.m1198

5. Roy S, Vallepu S, Barrios C, Hunter K (2018) Comparison of comorbid conditions between cancer survivors and age-matched patients without cancer. J Clin Med Res. 10:911-9. https://doi.org/ $10.14740 /$ jocmr $3617 \mathrm{~W}$

6. Isidori A, de Leval L, Gergis U, Musto P, Porcu P (2020) Management of patients with hematologic malignancies during the COVID-19 pandemic: practical considerations and lessons to be learned. Front Oncol 10:1439. https://doi.org/10.3389/fonc.2020. 01439

7. Vijenthira A, Gong IY, Fox TA, Booth S, Cook G, Fattizzo B, Martín-Moro F, Razanamahery J, Riches JC, Zwicker J, Patell R, Vekemans MC, Scarfò L, Chatzikonstantinou T, Yildiz H, Lattenist R, Mantzaris I, Wood WA, Hicks LK (2020) Outcomes of patients with hematologic malignancies and COVID-19: a systematic review and meta-analysis of 3377 patients. Blood 136:2881-2892. https://doi.org/10.1182/blood.2020008824

8. Ministry of Health \& family Welfare. Clinical management protocol: COVID-19 version 5 [Internet]. Mohfw.gov.in (web archive link, 23 October 2020). 2020 [cited 23 October 2020]. Available from: https://www.mohfw.gov.in/pdf/UpdatedClinicalManag ementProtocolforCOVID-19dated03072020.pdf

9. Salunke AA, Nandy K, Pathak SK et al (2020) Impact of COVID -19 in cancer patients on severity of disease and fatal outcomes: a systematic review and meta-analysis. Diabetes Metab Syndr 14:1431-1437. https://doi.org/10.1016/j.dsx.2020.07.037
10. Desai A, Sachdeva S, Parekh T, Desai R (2020) Covid-19 and cancer: lessons from a pooled metaanalysis. JCO Glob Oncol 6:557-559. https://doi.org/10.1200/GO.20.00097

11. ElGohary GM, Hashmi S, Styczynski J, Kharfan-Dabaja MA, Alblooshi RM, de la Cámara R, Mohmed S, Alshaibani A, Cesaro S, Abd El-Aziz N, Almaghrabi R, Gergis U, Majhail NS, El-Gohary Y, Chemaly RF, Aljurf M, El Fakih R (2020) The risk and prognosis of COVID-19 infection in cancer patients: a systematic review and meta-analysis. Hematol Oncol Stem Cell Ther 30:S1658-3876(20)30122-9. https://doi.org/10.1016/j.hemonc. 2020.07.005

12. Afshar ZM, Dayani M, Naderi M, Ghanbarveisi F, Shiri S, Rajati F (2020) Fatality rate of COVID-19 in patients with malignancies: a sytematic review and meta-analysis. J Infect 81:e114-e116. https://doi.org/10.1016/j.jinf.2020.05.062

13. Patel R, Park J, Shah A, Saif MW (2020) COVID-19 and cancer patients. Cancer Med J 3:40-48

14. He W, Chen L, Chen L, Yuan G, Fang Y, Chen W, Wu D, Liang B, Lu X, Ma Y, Li L, Wang H, Chen Z, Li Q, Gale RP (2020) COVID-19 in persons with haematological cancers. Leukemia 34:1637-1645. https://doi.org/10.1038/s41375-020-0836-7

15. Borah P, Mirgh S, Sharma SK, Bansal S, Dixit A, Dolai TK, Lunkad S, Gupta N, Singh G, Jain A, Bansal D, Choudhary D, Khandelwal V, Doval D, Kumar M, Bhargava R, Chakrabarti A, Kalashetty M, Rauthan A, Kazi B, Mandal PK, Jeyaraman P, Naithani R, AIIMS Hematology Alumni Group (2021) Effect of age, comorbidity and remission status on outcome of COVID-19 in patients with hematological malignancies. Blood Cells Mol Dis $87: 102525$

16. Foà R, Bonifacio $\mathrm{M}$, Chiaretti $\mathrm{S}$, Curti $\mathrm{A}$, Candoni $\mathrm{A}$, Fava $\mathrm{C}$, Ciccone M, Pizzolo G, Ferrara F (2020) Philadelphia-positive acute lymphoblastic leukaemia (ALL) in Italy during the COVID19 pandemic: a Campus ALL study. Br J Haematol 190:e3-e5. https://doi.org/10.1111/bjh.16758

17. Cuneo A, Scarfò L, Reda G, Varettoni M, Quaglia FM, Marchetti M, De Paoli L, Re F, Pietrasanta D, Rigolin GM, Orsucci L, Ibatici A, Gattei V, Mauro FR, Trentin L, Laurenti L, Marasca R, Foà R (2020) Chronic lymphocytic leukemia management in Italy during the COVID-19 pandemic: a Campus CLL report. Blood 136:763-766. https://doi.org/10.1182/blood.2020006854

18. Breccia M, Abruzzese E, Bocchia M, Bonifacio M, Castagnetti F, Fava C, Galimberti S, Gozzini A, Gugliotta G, Iurlo A, Latagliata R, Luciano L, Pregno P, Rege-Cambrin G, Rosti G, Stagno F, Tiribelli M, Foà R, Saglio G, Campus CML working group (2020) Chronic myeloid leukemia management at the time of the COVID-19 pandemic in Italy. A campus CML survey. Leukemia 34:2260-2261. https://doi.org/10.1038/s41375-020-0904-z

19. Wood WA, Neuberg DS, Thompson JC, Tallman MS, Sekeres MA, Sehn LH, Anderson KC, Goldberg AD, Pennell NA, Niemeyer CM, Tucker E, Hewitt K, Plovnick RM, Hicks LK (2020) Outcomes of patients with hematologic malignancies and COVID19: a report from the ASH Research Collaborative Data Hub. Blood Adv 4:5966-5975. https://doi.org/10.1182/bloodadvances. 2020003170

20. Lee KA, Ma W, Sikavi DR, Drew DA, Nguyen LH, Bowyer RCE, Cardoso MJ, Fall T, Freidin MB, Gomez M, Graham M, Guo CG, Joshi AD, Kwon S, Lo CH, Lochlainn MN, Menni C, Murray B, Mehta R, Song M, Sudre CH, Bataille V, Varsavsky T, Visconti A, Franks PW, Wolf J, Steves CJ, Ourselin S, Spector TD, Chan AT, COPE consortium (2021) Cancer and risk of COVID-19 through a general community survey. Oncologist 26:2020-36. https://doi. org/10.1634/theoncologist.2020-0572

21. Liang W, Guan W, Chen R, Wang W, Li J, Xu K, Li C, Ai Q, Lu W, Liang H, Li S, He J (2020) Cancer patients in SARS-CoV-2 infection: a nationwide analysis in China. Lancet Oncol 21:335337. https://doi.org/10.1016/S1470-2045(20)30096-6 
22. Lattenist R, Yildiz H, De Greef J, Bailly S, Yombi JC (2020) COVID-19 in adult patients with hematological disease: analysis of clinical characteristics and outcomes. Indian J Hematol Blood Transfus 37:1-5. https://doi.org/10.1007/s12288-020-01318-4

23. Shah V, KoKo T, Zuckerman M, Vidler J, Sharif S, Mehra V, Gandhi S, Kuhnl A, Yallop D, Avenoso D, Rice C, Sanderson R, Sarma A, Marsh J, de Lavallade H, Krishnamurthy P, Patten P, Benjamin R, Potter V, Ceesay MM, Mufti GJ, Norton S, Pagliuca A, Galloway J, Kulasekararaj AG (2020) Poor outcome and prolonged persistence of SARS-CoV-2 RNA in COVID-19 patients with haematological malignancies; King's College Hospital experience. Br J Haematol 190:e279-e282. https://doi.org/10.1111/bjh. 16935

24. Dai M, Liu D, Liu M, Zhou F, Li G, Chen Z, Zhang Z, You H, Wu M, Zheng Q, Xiong Y, Xiong H, Wang C, Chen C, Xiong F, Zhang Y, Peng Y, Ge S, Zhen B, Yu T, Wang L, Wang H, Liu Y, Chen Y, Mei J, Gao X, Li Z, Gan L, He C, Li Z, Shi Y, Qi Y, Yang J, Tenen DG, Chai L, Mucci LA, Santillana M, Cai H (2020) Patients with cancer appear more vulnerable to SARS-CoV-2: a multicenter study during the COVID-19 outbreak. Cancer Discov 10:783-791. https://doi.org/10.1158/2159-8290.CD-20-0422

25. Passamonti F, Cattaneo C, Arcaini L, Bruna R, Cavo M, Merli F, Angelucci E, Krampera M, Cairoli R, Della Porta MG, Fracchiolla N, Ladetto M, GambacortiPasserini C, Salvini M, Marchetti M, Lemoli R, Molteni A, Busca A, Cuneo A, Romano A, Giuliani N, Galimberti S, Corso A, Morotti A, Falini B, Billio A, Gherlinzoni F, Visani G, Tisi MC, Tafuri A, Tosi P, Lanza F, Massaia M, Turrini M, Ferrara F, Gurrieri C, Vallisa D, Martelli M, Derenzini E, Guarini A, Conconi A, Cuccaro A, Cudillo L, Russo D, Ciambelli F, Scattolin AM, Luppi M, Selleri C, Ortu La Barbera E, Ferrandina C, Di Renzo N, Olivieri A, Bocchia M, Gentile M, Marchesi F, Musto P, Federici AB, Candoni A, Venditti A, Fava C, Pinto A, Galieni P, Rigacci L, Armiento D, Pane F, Oberti M, Zappasodi P, Visco C, Franchi M, Grossi PA, Bertù L, Corrao G, Pagano L, Corradini P, ITA-HEMA-COV Investigators (2020) Clinical characteristics and risk factors associated with COVID-19 severity in patients with haematological malignancies in Italy: a retrospective, multicentre, cohort study. Lancet Haematol 7:e737-e745. https://doi.org/10.1016/S2352-3026(20)30251-9

26. Li X, Geng M, Peng Y, Meng L, Lu S (2020) Molecular immune pathogenesis and diagnosis of COVID-19. J Pharm Anal 10:102108. https://doi.org/10.1016/j.jpha.2020.03.001

27. García-Suárez J, de la Cruz J, Cedillo Á, Llamas P, Duarte R, Jiménez-Yuste V, Hernández-Rivas JÁ, Gil-Manso R, Kwon M, Sánchez-Godoy P, Martínez-Barranco P, Colás-Lahuerta B, Herrera P, Benito-Parra L, Alegre A, Velasco A, Matilla A, AláezUsón MC, Martos-Martínez R, Martínez-Chamorro C, SusanaQuiroz K, Del Campo JF, de la Fuente A, Herráez R, Pascual A, Gómez E, Pérez-Oteyza J, Ruiz E, Alonso A, González-Medina J, Martín-Buitrago LN, Canales M, González-Gascón I, VicenteAyuso MC, Valenciano S, Roa MG, Monteliu PE, López-Jiménez J, Escobar CE, Ortiz-Martín J, Diez-Martin JL, Martinez-Lopez J, AsociaciónMadrileña de Hematología y Hemoterapia (AMHH) (2020) Impact of hematologic malignancy and type of cancer therapy on COVID-19 severity and mortality: lessons from a large population-based registry study. J Hematol Oncol 13:133. https:// doi.org/10.1186/s13045-020-00970-7

28. Yigenoglu TN, Ata N, Altuntas F, Basc1 S, Dal MS, Korkmaz S, Namdaroglu S, Basturk A, Hacibekiroglu T, Dogu MH, Berber İ, Dal K, Erkurt MA, Turgut B, Ulgu MM, Celik O, Imrat E, Birinci S (2021) The outcome of COVID-19 in patients with hematological malignancy. J Med Virol 93:1099-1104. https://doi.org/ 10.1002/jmv.26404

Publisher's note Springer Nature remains neutral with regard to jurisdictional claims in published maps and institutional affiliations. 